\title{
CONSISTENCY IMPROVEMENT IN COMBINATORY SPANNING TREE ENUMERATION METHOD
}

\author{
Sergey Kadenko \\ Institute for Information Recording \\ of the National Academy of Sciences of Ukraine \\ Kiev, Ukraine \\ E-mail: sergeykadenko@mail.ru
}

\begin{abstract}
The paper addresses the problem of consistency improvement in group decision-making. The research is done in the context of studies performed by the Laboratory for Decision Support Systems of IIR of NASU (http://dss-lab.org.ua/). Particularly, the paper focuses on the problem of improving the consistency of pair comparison matrices (PCM) in AHP-based group decision support method called "enumeration of all spanning trees" or "combinatory algorithm". Quite often expert judgments do not satisfy consistency requirements. PCM provided by an individual expert can be inconsistent within itself, while matrices built by several experts in the context of the same decision-making procedure can be mutually inconsistent. Combinatory methods of expert judgment aggregation are designed to utilize the redundancy of expert data most thoroughly. But such aspects as satisfactory PCM consistency level and ways of consistency improvement still need to be studied more carefully. The task, tackled in the current paper, is to study the opportunities for development of a converging consistency improvement procedure, allowing to achieve satisfactory levels of initially inconsistent expert judgments in combinatory aggregation methods.
\end{abstract}

Keywords: pair comparison matrix, expert judgment consistency, enumeration of all spanning trees, combinatory algorithm 


\section{Introduction}

Expert data-based decision making is used mostly in weakly structured subject domains. In such domains it is problematic to perform quantitative measurements of these or that indicators influencing particular decisions. Moreover, there are no yardstick values which could be used for reference when expert evaluation is performed. For these reasons, expert data can be the only source of information under the abovementioned circumstances. But for these same reasons, expert data is characterized by inconsistency. Inconsistency can be witnessed in both ordinal and cardinal expert judgments. Also, inconsistency characterizes both individual and group expert estimates. Consequently, consistency considerations must be taken into account in every decision-making support method where expert data is used. Particularly, the questions to be addressed are: "how can consistency of expert judgments be measured in a given method?"; "what is the satisfactory expert judgment consistency level when a certain number of objects is evaluated (compared) by a certain number of experts?" and "how can expert judgment consistency level be improved, if necessary?" Or, in other words, "what is the borderline between redundancy of expert information (considered a good feature) and inconsistency of expert information, and how is it crossed?"

This particular paper focuses on consistency considerations in the context of AHP-based combinatory method of enumeration of all spanning trees (see Tsyganok (2010), Mikhailov/Siraj/Keane (2012)). The method fully utilizes the redundancy of expert information in group and individual decision-making, but consistency of expert judgments and its improvement procedures require further study.

\section{Literature Review}

Combinatory method was first suggested by V.Tsyganok in early 2000-s - respective publication appeared in "Mathematical Modelling" journal in 2010 (Tsyganok (2010)). A few years later a very similar approach was suggested by Siraj\Mikhailov \Keane (2012). As for consistency in the context of AHP and related methods, the following studies should be mentioned: Iida studied ordinal consistency improvement in AHP through elimination of circular triads (details can be found in his paper from ISAHP 2009). Mikhailov and Siraj, again, studied ordinal consistency improvement (details can be found in their paper from ISAHP 2011). Brunelli and Fedrizzi in their paper from ISAHP 2011 analyzed several consistency indicators in AHP but did not suggest any particular consistency improvement methods. Mikhailov/Siraj/Keane (2012) do not suggest any feedback procedure when PCM are not consistent enough. Saaty (1996) himself does not prescribe any particular methods for consistency improvement if consistency index values are unsatisfactory: in such cases he just recommends to "revise the judgments and reconsider the problem". The nature of consistency indices in AHP (CR, CI, RI) does not provide for particular consistency improvement steps to be taken (particular objects swapped in rankings or PCM, or particular experts to be re-addressed with suggestions to change their judgments in order to improve their consistency). Tsyganok (2010) uses spectral consistency coefficient suggested by Totsenko (1996). Consistency improvement mechanism based on this coefficient is somewhat similar to Delphi approach (experts whose judgments lie outside the "majority" area are asked to change their judgments accordingly), but it is more target-oriented and flexible. Utilization of spectral consistency coefficient as consistency measure in combinatory algorithm allows to tell, 
which expert must be addressed with a suggestion to change his judgment, and which particular judgment (pair comparison) must be changed. On the other hand, the spectral coefficient is not devoid of certain drawbacks: firstly, it heavily depends on the scale point size, and, secondly, it unites two indicators - dispersion and entropy, which are, in the general case, independent. Consistency improvement procedure used by Tsyganok is not monotonously convergent, although its testing on multiple examples indicates that sufficient consistency level (Totsenko (1996) called it "usability threshold" or $T_{u}$ ) can be achieved. It would be adequate to try developing a monotonously convergent procedure for expert judgment consistency improvement during combinatory algorithm-based aggregation of individual PCM.

\section{Hypotheses/Objectives}

The problem to be addressed can be formulated as follows. Let us say, a certain number of experts estimates a certain number of objects (or decision variants) according to a certain criterion. Aggregation of individual PCM into a group PCM is performed using the method of enumeration of all spanning trees (or combinatory method). The task is to study the opportunities for devising a monotonously convergent procedure allowing to achieve expert judgment consistency level, which is sufficient for aggregation of individual PCM into a group one. I.e., the procedure must show which expert must be addressed with suggestions to change the judgments and which particular pair comparisons must be changed in the first place to improve the overall consistency level.

\section{Research Design/Methodology}

The first thing to be kept in mind is that there are several aspects of consistency to be tackled: ordinal versus cardinal consistency and inner versus mutual PCM consistency. The case can be illustrated by an example of an orchestra where each instrument is perfectly tuned to a different pitch. Inner consistency of pair comparisons does not guarantee mutual consistency of individual pair comparison matrices.

Approaches to ordinal consistency improvement within pair comparison matrices were suggested by Mikhailov, particularly, in his paper from ISAHP 2011. If the matrix is ordinally consistent, its elements can be rearranged in such a way that all elements above the principal diagonal are positive. Again, if we need to get a consistent set of pair comparison matrices, built by several experts, the order of alternatives in all experts' rankings needs to be the same, and this condition is the most problematic to fulfill (experts may be reluctant to swap ranks of alternatives), and there is no clear mutual ordinal consistency improvement algorithm. However, there are some rules which can be followed (see Tsyganok/Kadenko (2012)).

If the matrix is ordinally consistent within itself, all the elements lying below the principal diagonal are less than 1 (alternatives can be rearranged in such a way). When such matrices are obtained, spanning tree enumeration method (Tsyganok 2010, Mikhilov/Siraj/Keane 2012) can be launched. As a result, we obtain an aggregate PCM. Based on this matrix alternative weights can be calculated (using eigenvecor or some other method). 
But if consistency level is not considered sufficient enough (respective PCM elements are considered "too different"), it is appropriate to shift the respective element of individual pair comparison matrix towards the element of the aggregate matrix, to improve consistency level. The first element to shift (to suggest to an expert for a change) would be the element, which differs from respective aggregate matrix element most significantly. The size of the shift can constitute half of this difference (to ensure better fine-tuning of respective matrix elements).

If we allow the elements of pair comparison matrices to assume values not only from fundamental scale $(1 / 9, \ldots, 1 / 2,1,2, \ldots, 9)$, but all the values within the range, then the consistency improvement procedure can allow to make differences between pair comparison matrices (individual ones and aggregate one) as small as it is required. Thus, we shall have a monotonously converging consistency improvement procedure.

\section{Data/Model Analysis}

In this section we shall analyze a simple numerical consistency improvement example, where 3 experts estimate 4 objects, and aggregate preference matrix is built using the combinatory method (Table 1 ).

Table 1. Initial pair comparison matrices

\begin{tabular}{|l|l|l|l|l|l|l|l|l|l|l|l|l|l|r|r|r|r|}
\hline & Expert 1 & \multicolumn{1}{|l|}{ Expert 2 } & \multicolumn{3}{|c|}{ Expert 3 } & \multicolumn{4}{|c|}{ Aggregate matrix } \\
\hline A1 & 1.00 & 3.00 & 4.00 & 8.00 & 1.00 & 2.00 & 4.00 & 9.00 & 1.00 & 2.00 & 4.00 & 8.00 & 1 & 2.14 & 4.14 & 8.26 \\
\hline A2 & 0.33 & 1.00 & 2.00 & 5.00 & 0.50 & 1.00 & 2.00 & 4.00 & 0.50 & 1.00 & 2.00 & 3.00 & 0.47 & 1 & 1.93 & 3.86 \\
\hline A3 & 0.25 & 0.50 & 1.00 & 2.00 & 0.25 & 0.50 & 1.00 & 2.00 & 0.25 & 0.50 & 1.00 & 2.00 & 0.24 & 0.52 & 1 & 2.00 \\
\hline A4 & 0.13 & 0.20 & 0.50 & 1.00 & 0.11 & 0.25 & 0.50 & 1.00 & 0.13 & 0.33 & 0.50 & 1.00 & 0.12 & 0.26 & 0.50 & 1 \\
\hline
\end{tabular}

The largest difference from respective aggregate matrix is witnessed in element $a_{24}$ of the matrix built by Expert 1. Consequently, it is this element that needs to be changed (offered to Expert 1 for change) in the first place. If we permit to use real numeric values, and not only integer ones from fundamental scale, the picture after the $1^{\text {st }}$ iteration will look as follows (Table 2).

Table 2. Consistency improvement: iteration 1 (real values)

\begin{tabular}{|c|c|c|c|c|c|c|c|c|c|c|c|c|c|c|c|c|}
\hline & \multicolumn{4}{|c|}{ Expert 1} & \multicolumn{4}{|c|}{ Expert 2} & \multicolumn{4}{|c|}{ Expert 3} & \multicolumn{4}{|c|}{ Aggregate matrix } \\
\hline $\mathrm{A} 1$ & 1.00 & 3.00 & 4.00 & 8.00 & 1.00 & 2.00 & .00 & 9.00 & 1.00 & 2.00 & 4.00 & 8.00 & 1 & 2.17 & 4.14 & 8.20 \\
\hline $\mathrm{A} 2$ & 0. & 1.0 & 2.00 & 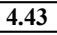 & 0.5 & 1. & 2.00 & 4.0 & 0.50 & 1.00 & 2.0 & 3.00 & 0.46 & 1 & 1.91 & 3.79 \\
\hline $\mathrm{A} 3$ & 0.25 & 0.5 & 1.00 & 2.00 & 0.2 & 0.5 & 1.00 & 2.0 & 0.25 & 0.50 & 1.0 & 2.00 & 0.24 & .52 & 1 & 1.98 \\
\hline $\mathrm{A} 4$ & 0.13 & 0.23 & 0.50 & 1.00 & 0. & 0.25 & 0.50 & 1.0 & 0.1 & 0.33 & 0.50 & 1.00 & 0.1 & 0.26 & 0.51 & 1 \\
\hline
\end{tabular}

If real values are not permitted, the picture after the $1^{\text {st }}$ iteration will look as follows (Table 3).

Table 3. Consistency improvement: iteration 1 (integer fundamental scale values)

\begin{tabular}{|c|c|c|c|c|c|c|c|c|c|c|c|c|c|c|c|c|}
\hline & \multicolumn{4}{|c|}{ Expert 1} & \multicolumn{4}{|c|}{ Expert 2} & \multicolumn{4}{|c|}{ Expert 3} & \multicolumn{4}{|c|}{ Aggregate matrix } \\
\hline $\mathrm{A} 1$ & 1.00 & 3.00 & 4.00 & 8.00 & 1.00 & 2.00 & 4.00 & 9.00 & 1.00 & 2.00 & 4.00 & 8.00 & 1 & 2.19 & 4.15 & 8.15 \\
\hline $\mathrm{A} 2$ & 0 & 1.00 & & & & 100 & & & & 1.00 & & & 46 & 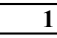 & 1.90 & 3.73 \\
\hline $\mathrm{A} 3$ & 0.2 & 0. & 1.0 & 2.0 & 0. & 0.5 & 1.0 & 2. & & 0.5 & 1. & 2.0 & 4 & .53 & 1 & 1.97 \\
\hline$\overline{\mathrm{A} 4}$ & 0.13 & 0.23 & 0.50 & 1.00 & 0.11 & 0.25 & 0.50 & 1.00 & 0.13 & 0.33 & 0.50 & 1.00 & 0.12 & 0.27 & 0.51 & 1 \\
\hline
\end{tabular}

Following the pattern of changing individual pair comparisons, which are most considerably differing from respective aggregate ones at every new iteration, we will get a thoroughly consistent individual matrices and aggregate matrix (Table 4)
International Symposium of 
Table 4. Thoroughly consistent matrix

$\begin{array}{cccc}1 & 2 & 4 & 8 \\ 0.5 & 1 & 2 & 4 \\ 0.25 & 0.5 & 1 & 2 \\ 0.13 & 0.25 & 0.5 & 1\end{array}$

It should be noted that in case if real values are permitted, the procedure is monotonously convergent.

\section{Limitations}

In reality experts are not operating with real values, they are only presented verbal scales with respective integer number equivalents. Consequently, in a real expert examination it is, virtually, impossible to achieve ideal consistency of pair comparison matrices (both mutual and inner). The procedure of consistency improvement should, definitely, stop when the absolute value of difference between individual and aggregate pair comparisons start to increase with the new iteration. It should be also noted that prior to launching the described consistency improvement procedure, ordinal consistency of pair comparison matrices should be ensured.

\section{Conclusions}

Several aspects of pair comparison consistency improvement in combinatorial aggregation method have been analyzed. Based on the analyses, a monotonously convergent consistency improvement procedure has been suggested. The suggested approach has its limitations, namely, in order for procedure to converge, it requires ordinal consistency of individual pair comparison matrices. However, the approach can be utilized as a consistency improvement method in group decision-making (including AHP/ANP-based decision-making).

\section{Key References}

Kadenko,S., Tsyganok, V. (2012). A Method for Improving the Consistency of Individual Expert Rankings during Their Aggregation. Journal of Automation and Information Sciences, 44(4), 23-31.

Saaty, T.L. (1996). Decision Making with Dependence and Feedback: The Analytic Network Process. Pittsburgh, PA: RWS Publications.

Siraj S., Mikhailov L., Keane J. (2012). Enumerating All Spanning Trees for Pairwise Comparisons. Computers and Operations Research, 39(2), 191-199.

Totsenko, V.G. (1996). The Agreement Degree of Estimations Set with Regard for Experts' Competence. Proceedings of the IV International Symposium on the AHP, ISAHP '96. Simon Fraser University, Burnaby, B.C.,CANADA, 229-241.

Tsyganok, V. (2010). Investigation of the aggregation effectiveness of expert estimates obtained by the pairwise comparison method. Mathematical Modeling, 52(3-4), 538-544. 